\title{
Protective role of NGAL in ANCA-induced glomerulonephritis
}

\section{C6 \\ IL-17A has \\ an important \\ role in the development \\ of AAV and \\ associated \\ kidney damage}

Neutrophils are directly activated by antineutrophil cytoplasmic antibodies (ANCAs) and have an important role in the development of vascular inflammation and kidney damage in patients with ANCA-associated vasculitis (AAV). Now, Adrian Schreiber and colleagues report that neutrophil gelatinase-associated lipocalin (NGAL) is released by ANCA-stimulated neutrophils and might have a protective role in AAV.

"NGAL is expressed in kidney tubular cells and is released into the urine by these cells during acute kidney injury. For this reason, NGAL has been used as a marker of kidney tubular cell damage in several kidney diseases," comments Schreiber. "In parallel, growing evidence suggests that NGAL expressed by myeloid cells - particularly neutrophils, which have huge amounts of NGAL stored in their granules - could modulate inflammation during bacterial infection and in autoimmune diseases. However, the exact role of NGAL remains controversial, as NGAL deletion has been associated with both beneficial and detrimental effects in mice with multiple sclerosis and glomerulonephritis."

Schreiber and colleagues report that serum and urine levels of NGAL were increased in patients with active AAV compared with healthy individuals,

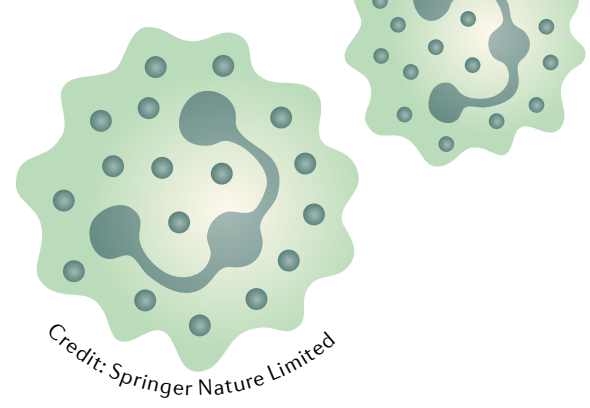

patients with inactive AAV and patients with lupus nephritis. Similarly, serum and urine NGAL levels were significantly higher in a mouse model of antimyeloperoxidase (anti-MPO) ANCA-induced necrotizing crescentic glomerulonephritis (NCGN) than in healthy controls.

"Based on our observation that ANCA-stimulated neutrophils release a huge amount of NGAL in vitro, we hypothesized that ANCA-activated neutrophils that infiltrate the kidney release NGAL and might modulate the local inflammatory response," says Schreiber. To investigate this hypothesis, the researchers used their anti-MPO ANCA mouse model of NCGN.

"First we immunized MPOdeficient mice with murine MPO," explains Schreiber. "After the mice had developed anti-MPO antibodies, we irradiated them and then transplanted them with bone marrow cells from either wild-type or NGAL-deficient mice to generate chimeric $\mathrm{Mpo}^{-/-}$ wild-type and $\mathrm{Mpo}^{-/-} / \mathrm{Ngal}^{-/-}$mice. Although both groups of chimeric mice developed NCGN, we observed that specific deletion of NGAL in haematopoietic cells, including myeloid cells, worsened kidney damage in our model." Neutrophil function was similar between the chimeric NGAL-deficient and wild-type mice, suggesting

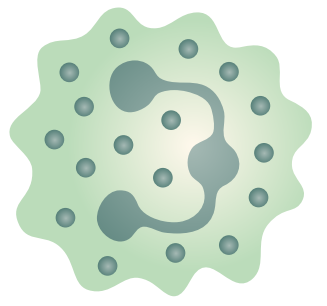

that NGAL does not protect against kidney damage in AAV by downregulating neutrophil function. However, the NGAL-deficient mice showed increased levels of kidney-infiltrating $\mathrm{CD}^{+} \mathrm{T}$ cells, particularly $\mathrm{T}$ helper 17 $\left(\mathrm{T}_{\mathrm{H}} 17\right)$ cells, compared with the wild-type controls. Splenocytes from chimeric NGAL-deficient mice produced more IL-17A than those from wild-type controls, and the biologically active form of NGAL, iron siderophore-loaded NGAL, inhibited splenocyte $\mathrm{T}_{\mathrm{H}} 17$ cell polarization in vitro, suggesting that NGAL might downregulate $\mathrm{T}_{\mathrm{H}} 17$ cells.

Mice with genetic deletion of both MPO and IL-17A $\left(\mathrm{Mpo}^{-/-} / I l 17 \mathrm{a}^{-/-}\right)$ that were immunized with MPO and then transplanted with bone marrow cells from $\mathrm{Ngal}^{-/-} / \mathrm{Ill} \mathrm{a}^{-/-}$mice were protected against the development of NCGN compared with $\mathrm{Mpo}^{-/-}$ mice that were transplanted with $\mathrm{Ngal}^{-/-}$bone marrow cells. "These findings confirm that IL-17A has an important role in the development of AAV and associated kidney damage," says Schreiber.

The researchers conclude that bone-marrow-derived NGAL, most likely produced by neutrophils, protects against kidney damage in AAV by downregulating $\mathrm{T}_{\mathrm{H}} 17$ immunity. "Neutrophils are generally thought to be 'the bad guys' in AAV," says Schreiber. "Our new data show that neutrophils might also release protective mediators, such as NGAL, and suggest that they could modulate and suppress T cell responses."

Ellen F. Carney

ORIGINAL ARTICLE Schreiber, A. et al. Neutrophil gelatinase-associated lipocalin protects from ANCA-induced GN by inhibiting $\mathrm{T}_{\mathrm{H}} 17$ immunity. J. Am. Soc. Nephrol. https://doi.org/ 10.1681/ASN.2019090879 (2020) 